This item was submitted to Loughborough's Research Repository by the author.

Items in Figshare are protected by copyright, with all rights reserved, unless otherwise indicated.

\title{
Optimal charging of EVs in a real time pricing electricity market
}

PLEASE CITE THE PUBLISHED VERSION

https://doi.org/10.4271/2013-01-1445

PUBLISHER

(C) SAE International

VERSION

AM (Accepted Manuscript)

PUBLISHER STATEMENT

Copyright (C) 2013 SAE International. This paper is posted on this site with permission from SAE International. It may not be shared, downloaded, transmitted in any manner, or stored on any additional repositories or retrieval system without prior written permission from SAE.

\section{LICENCE}

All Rights Reserved

\section{REPOSITORY RECORD}

Mody, Sagar, and Thomas Steffen. 2015. "Optimal Charging of Evs in a Real Time Pricing Electricity Market". figshare. https://hdl.handle.net/2134/17430. 


\title{
Optimal Charging of EVs in a Real Time Pricing Electricity Market
}

\author{
Sagar Mody, Thomas Steffen \\ Loughborough University
}

Copyright $\odot 2012$ SAE International

\begin{abstract}
$\underline{\text { ABSTRACT }}$
The idea of grid friendly charging is to use electricity from the grid to charge batteries when electricity is available in surplus and cheap. There are several ways of achieving this, for example using droop control, using night time electricity tariffs, or using smart metering. The goal is twofold: to avoid putting additional load on the electricity grid and power generation, and to reduce the cost to the consumer.
\end{abstract}

This paper looks at the saving potential when charging an electric car using real time tariffs provided by a smart meter, using the Ameren tariffs in Illinois as an example. If prices are known in advance (day-ahead pricing), the optimization only requires picking the cheapest time slots for charging the battery. Further savings can be made by using real time prices that are not known in advance, but the optimization problem then depends on price prediction models, and it becomes much more difficult to solve. This paper presents a simple suboptimal approach, and it quantifies the potential improvements that could be made using more sophisticated price predictions.

The result is that cost savings in the order of about 50 USD (1/3 of the electricity costs) are feasible if a fast charger is used using real time pricing. The scale of the savings is such that complex optimization strategies are not worthwhile, and for the foreseeable future simple solutions are expected to be more cost effective.

\section{INTRODUCTION}

The power and transport sectors are the first and second largest sources of global greenhouse gas (GHG) emissions. The transport sector contributions to both emissions and energy use are growing quickly by the year and World Energy Outlook projections predict that they will overtake the power sector by 2035 [1][2]. In 2007, the road transport sector accounted for $71 \%$ of the total emissions attributed to the sector as a whole, with $63 \%$ of them generated by passenger cars [3]. The world community has set ambitious targets for GHG reduction in the future and many countries (especially the developed nations) have registered emission reduction targets or commitments to the actions by 2020. As an example, the European Union's (EU) ambitious target is set for 2020 to reduce $\mathrm{GHG}$ emissions by at least $20 \%$, improve energy efficiency by $20 \%$ and ensure the contribution of renewable energy sources in gross energy consumption is $20 \%$ [4].

\section{Electric Vehicles}

The problem of climate change has been demanding cleaner and more energy-efficient powertrains, for over two decades. However, the recent route towards 'greener environment' policies has brought electric vehicles $(\mathrm{EV})$ to attention and $\mathrm{EV}$ research to the forefront of many original equipment manufacturers' (OEM) future plans.

1. Hybrid electric vehicles (HEV) are a combination of the typical internal combustion engine (ICE) and a battery electric vehicle (BEV), with the electric motor supplying auxiliary power when the ICE is not in use. The ICE is able to recharge the batteries when the EV is not in use and the on board computer manages the correct mix of electric and fuel power depending on the engine and battery power available. The ICE essentially works as a range extender while the urban battery use reduces overall emissions.

2.BEVs are purely battery powered and use a motor or combination of motors to drive the vehicle. BEVs suffer from limited driving range because of battery limits. However, research in battery technology is rigorous in the current environment and is bringing many improvements in range and battery life. Currently, lithium-ion (Li-ion) batteries are the most promising battery technology with the ability to store significantly more electricity in much lighter cell packs.

3. Plug-in hybrid vehicles (PHEV) combine the advantages of the HEV and BEV. They work in two modes: fully electric or hybrid. The ICE can facilitate the drive or function individually, just like the BEV. The other advantage this technology has is that it can be charged using a wall socket just like BEVs thus being able to have electric power for most urban trips without engaging the ICE at all. Currently, the pure electric drive mileage of

Page 1 of 13 
PHEVs is limited to 10 and 60 miles depending on battery size.

HEV have been mildly successful in the past decade with the major producer, Toyota (Prius) selling 2 million units by 2009 . Whilst it is widely accepted that market forces alone have not been able to make the EV a first choice for many consumers; government policy support, research to make EVs less expensive and economy improvement will improve their market significantly [5][6]. The barriers in EV penetration into the main market have been limited driving range and high cost of electric technology. Although, electric driving ranges are limited for all three HEV, PHEV and BEVs; surveys have indicated that $47-55 \%$ of single vehicle usage in a single day is less than 20 miles, with $82-88 \%$ of vehicles travelling less than 60 miles [7]. Kang and Recker's 2009 [7] study concludes that it is possible to convert between $80 \%$ to $90 \%$ of daily mileage to electric when using PHEV with a 60 mile range in California; under the condition that both home and public place charging stations are in use. These numbers indicate that EVs are more feasible than previously thought. JP Morgan performed a study in 2009 which forecasted 11.28 million EVs worldwide by 2020 and $20 \%$ of the total cars sold in North America [8].

\section{Impact of Electric Vehicle Penetration}

Although, a change from ICEVs to EVs means a little change for consumers in context of refueling patterns; their main impact will be that on the already stressed electric grids. The batteries of these vehicles require long times and high power and currents for charging. For most domestic users, charging will take place overnight at their homes. Moreover, the tendency to plug the vehicle in as soon as they reach home is high. Other typical charging loads could be concentrated in office or public car parks depending on the actual use of the vehicle during the day. If the consumers charge the vehicles after every trip: for example, every time they reach office or a supermarket or come home and leave again, this would be of even higher concern to electricity peaks. Although, peak load times would be different in different parts of the world, depending on weather, some kind of load shifting to accommodate EV charging may be required.

The subsequent effect to consider is the logical change to electricity generation and retail or wholesale prices in the future. The higher peaks, consumption and addition of more renewable energy might change the prices in markets significantly. In most countries the industrial sectors are on wholesale electricity prices- buying electricity at lower rates during off-peak hours. There is a possibility for such 'spotmarkets' even for domestic electricity consumers as is case in Portugal, Germany, some parts of continental Europe and a few states in the USA. In such markets, the consumers are encouraged to shift their electricity usage to off-peak hours through high-price updates/alerts either hourly or daily. Advances in 'Smart-Grid' technology can allow this elastic behavior from households, helping them to reduce costs. The meter records hourly consumption and also alerts the consumers of the latest and future prices. This essentially ties in with the idea of 'Real-Time Pricing (RTP)' where prices change on an hourly basis as mentioned before. The smartgrid technology includes communication devices which allow real time consumption checking and communication to and from the consumers' meter. The smart grid is being promoted and provided to consumers in the U.S.A. for some time now. Electric utility providers in California, Colorado, Florida, Illinois, Indiana, Ohio, Texas, Washington and some other states have already been introducing smart grids to many customers. There is also a strong financial incentive being provided for both smart grid research and introduction via the Energy Independence Act of 2007 and the US Stimulus Package of 2009 [9]. This strongly indicates that smart grids and RTP are the future of electric pricing and management. The state of Illinois is a good example where RTP has been available to customers since 2003. The RTP programs have been successful in reducing the participating consumers' electric usage and bills and shifting usage to non-peak times of the day [10]. The two electricity providers which allow the choice of RTP are Amaren and ComEd. Amaren's Power Smart Pricing (PSP) and ComEd's Residential Real Time Pricing (RRTP) programs have reduced their peak demand in the range of $15 \%$ and achieved participant bill savings between $10-15 \%$ [10].

The above discussion shows that there are two problems to consider when EV penetration becomes high in the future. Firstly, it will be important to manage the electricity loads and peak demands due to user profile of EV charging. Some load may have to be shifted from peak afternoon and evening times in some manner either by persuading or enforcing the consumer to charge earlier or later. Secondly, charging the vehicle without control might also be a disadvantage for the consumer due to the possibility of a future with RTP for electricity. However both these problems can be looked as an opportunity for EVs. The flexibility of charging time can be looked as an advantage for load shifting opportunities and the daily mileage being enough for urban travel means times of charging can be varied. When smart-grid infrastructure is in place in the future, communication with it can not only allow automatic flexible charging but can also be used to provide electricity from the vehicle to grid (V2G).V2G technology can level any load fluctuations by supporting the grid and gives the opportunity to sell surplus electricity in the EV to national grids). Flexible charging by smart-grid communication can allow future EVs to access RTP for electricity and charge for the lowest cost.

Finally, the charging of electric vehicles has impact on the electricity grid on a number of time scales, ranging from the millisecond range to hours and days. The potential is there for all these effects to be beneficial to the grid: active inverters can absorb harmonics, they can apply droop control to enhance grid stability, and they can pick times for charging when excess electricity is available. However, very little progress has been made towards these goals so far, and

Page 2 of 13 
regulations often only aim to prevent harmful effects, not to leverage potential benefits. This paper concentrates on the slow time scale, and what kind of effects smart metering has on the optimal charging timing.

Table 1: Grid Effects by Timescale

\begin{tabular}{|l|l|l|l|}
\hline Time Scale & Significance & Function & Advantage \\
\hline $\begin{array}{l}\text { Very Fast } \\
\mathbf{0 . 1 - 1 0 m s}\end{array}$ & $\begin{array}{l}\text { Harmonics } \\
\text { and Noise }\end{array}$ & $\begin{array}{l}\text { Absorbs } \\
\text { Disturbances }\end{array}$ & $\begin{array}{l}\text { Improves } \\
\text { Local Power } \\
\text { Quality }\end{array}$ \\
\hline $\begin{array}{l}\text { Medium } \\
\mathbf{0 . 1 - 1 0 s}\end{array}$ & $\begin{array}{l}\text { Grid } \\
\text { Frequency } \\
\text { Stability }\end{array}$ & $\begin{array}{l}\text { Droop } \\
\text { Control }\end{array}$ & $\begin{array}{l}\text { Supports } \\
\text { Grid }\end{array}$ \\
\hline $\begin{array}{l}\text { Long } \\
\mathbf{0 . 1 - 1 0 h}\end{array}$ & $\begin{array}{l}\text { Battery } \\
\text { Charge and } \\
\text { Electricity } \\
\text { Prices }\end{array}$ & $\begin{array}{l}\text { Cost } \\
\text { Optimisation }\end{array}$ & $\begin{array}{l}\text { Reduces } \\
\text { Charge Cost }\end{array}$ \\
& & & \\
\hline
\end{tabular}

\section{LITERATURE REVIEW}

Studies on the effect of EV penetration to the electricity grid date back to the 1980s. In [11], Heydt discusses the effects on and of electric load management due to EV penetration. The study concludes that it is likely that charging will occur at peak demand times and some form of load management must be introduced to manage the additional EV charging load. Webster's review of electric infrastructure in the UK [12] concludes that in case of high EV penetration, it is likely that battery recharging times will coincide with peak electricity demand. Measures must be taken to avoid this although the electric networks can cope with the additional load. Both these authors consider the user profiles to be of primary impact on recharging times. In a much more recent study, Camus et al. [13] simulate a 2020 scenario of 2 million EVs in the Portuguese spot electricity market, considering different mixes of renewable power generation. They conclude that with low renewables and high cost, charging of EVs during peak times can lead to electricity prices of 17 Euro cents/KWh. This can be brought down to 7 Euro cents/KWh with off-peak charging and with higher renewables and low general costs, down to 5.6 Euro cents/KWh. Mahalik et al. [14] performed a simulation to realize EV impacts on the Illinois grid in 2020 and concluded that on-peak uncontrolled charging would require an additional $400 \mathrm{MW}$ unit to support the state's reserve margin. If off-peak and controlled charging is facilitated, no additional supporting grid would be needed. The additional electricity required could be provided by reducing the electricity exports Illinois makes. All the above research recognizes the problems related to on-peak charging in a high EV penetration scenario but no mathematical formulations are presented for optimal charging.

Acha et al. [15] present a time coordinated optimal power flow (TCOPF) tool for distribution networks to decide on load control approaches for EVs in the future. The algorithms concentrate on showing different charging strategies to the electricity providers to see how they may have to change energy production to reduce carbon emissions and cost. They conclude for the UK context that, UK will need to introduce more renewables or non-carbon fuel mix to offset costs and emissions for high EV charging scenarios. Kristoffersen et al. [16] use a linear regression to minimize charging costs based on the Danish (Norpool) electricity market prices. The study made the assumption of an EV fleet controller who managed the participation of EVs during charging or providing electricity to the grid, based on fleet driving patterns and electricity prices. They concluded that EV driving patterns and hence charging time is highly flexible during the day but not from day to day. In [17], Rotering and Ilic present two timediscrete algorithms for optimal charging; one considering only minimizing cost and the other also taking into account V2G support. The perform a case study based on the California day ahead electricity price market and conclude that optimal/smart charge reduces the charging cost from 0.43 USD to 0.2 USD daily. In case of V2G support, the profit amounts to 1.71 USD including charging.

The following paper presents a study on an optimal charging algorithm for future EVs which considers battery charging in a spot or real-time electricity market. The idea is to automatically manage the charging time once the vehicle is plugged in to provide a full charge when required but at the lowest cost. This means that the charging would be shifted to off-peak hours when the price is lower. The subsequent advantage of this can be to the grid in a way of load shifting. Firstly the optimal charging problem is explored based on time-discrete solution and then variability is introduced with the assumption that there might be power reduction at peak times. Secondly, the implication of introducing dynamics in the optimal solution achieved is looked at in the context of the unpredictability of the actual electric prices. A stochastic approach is discussed for the solution of the dynamic problem. Lastly, the example of Illinois RTP is taken and a possible scenario and case study for optimal charging is shown.

\section{THE BASIC OPTIMAL CHARGING PROBLEM}

A highly abstracted version of the optimal charging problem will be introduced first because this leads to a very simple solution that lends itself well to both implementation and further analysis. Additional details will be added and discussed afterwards.

The basic optimal charging problem is defined in discrete time with step size $T$. It has one control variable: the charging power $p_{k}$. The power is subject to two constraints: it cannot be negative and there is a constant maximum power $\bar{p}$ such that $p_{k} \in[0, \bar{p}]$. 
The behavior of the system is determined by two separate dynamics: the battery state and the total cost. Both accumulate (integrate) over time and the only difference is the coefficient.

The battery state $E_{k}$ is an integral of the charge power over time:

$$
E_{k+1}=E_{k}+T p_{k}
$$

assuming that the self-discharge and charging losses are negligible.

The total cost $C_{k}$ is also an integral of the charge power but weighted by the current electricity price: $C_{k+1}=C_{k}+T c_{k} p_{k}$

where $c_{k}$ denotes the electricity price and $C_{0}=0$. The price is a disturbance for the system, for now it is assumed that it is known in advance, which simplifies the problem significantly.

The total number of steps $l$ to consider with $k=0 \ldots l$ is also defined in advance.

Definition 1: The basic optimal charging problem.

The basic optimal charging problem is defined by the cost function $J=C_{l}$ representing the total electricity cost and the boundary condition $E_{l}=E_{\text {full }}$, which requires the battery to be fully charged at the end of the charging process.

Because no discharge is allowed, it is not necessary to impose limits on the charge state $E_{k}$, as it is already bounded by $E_{0}$ and $E_{\text {full }}$.

The advantage of using this simple model is that the final state and cost can easily be calculated as:

$$
\begin{aligned}
E_{l} & =E_{0}+\sum_{k=0 \ldots l-1} T p_{k} \\
C_{l} & =\sum_{k=0 \ldots l-1} T c_{k} p_{k}
\end{aligned}
$$

\section{Lemma:}

The solution to basic optimal charging problem is

$$
p_{k}^{*}=\left\{\begin{array}{c}
0, c_{k}>c^{\prime} \\
p^{\prime}, c_{k}=c^{\prime} \\
\bar{p}, c_{k}<c^{\prime}
\end{array}\right.
$$

The threshold price $c^{\prime}$ is one of the prices $c_{k}$ and the threshold charging power $p^{\prime}$ can be found from the boundary condition using a linear equation. There may be more than one solution if several time steps have the same price $c_{k}=c_{k^{\prime}}=c^{\prime}$ and for now it is assumed that is not the case.

\section{Proof:}

The proof has two parts. The first step is to demonstrate that the presented from is an admissible solution to the problem

Page 4 of 13 and the second is to show that it is indeed the/an optimal solution.

With the given control law, the final charge state $E_{l}$ is a function the initial state $E_{0}$, the threshold charging power $p^{\prime}$ and the number of full charging cycles $n$ that satisfy $c_{k}<c^{\prime}$. As long as $E_{l} \geq E_{0}$ and $E_{l} \leq E_{0}+l T \bar{p}$, the problem has a solution.

Assuming that the prices are different at each time step, there is exactly one solution, which is given by the following two equations:

$$
\begin{aligned}
& n=\text { floor } \frac{E_{l}-E_{0}}{T \bar{p}} \\
& p^{\prime}=\frac{E_{l}-E_{0}}{T}-n \bar{p}
\end{aligned}
$$

This solution is not just admissible but also optimal because any deviation from this solution within the charging power limits leads to a higher cost. In order to maintain the same final charge state $C_{l}$, an alternate solution needs to be decreased the charging power at some time step $p_{i}=p_{i}^{*}-$ $\Delta p$, and increased it at another $p_{j}=p_{j}^{*}+\Delta p$. This maintains the same integral and therefore satisfies the boundary condition. But increases are only admissible when $p_{j}<\bar{p}$, and decreases only when $p_{i}>0$. It follows that $c_{i} \leq c^{\prime} \leq c_{j}$, and therefore the net effect is an increase of charging cost by $\Delta J=T\left(c_{j}-c_{i}\right) \Delta p$. (qed)

\section{VARIATIONS OF THE BASIC CHARGING PROBLEM}

There are several extensions that can be made to make this problem more applicable. This section will list a number of variations that lead to essentially similar solutions. The basic approach is that the variation is converted back into the original form of the problem, so the same simple solution algorithm can be used.

\section{Varying Charge Power Limit}

The amount of available charging power $\bar{p}$ may change over time, for example to do electricity use restrictions at peak load periods. This means a new time series $\bar{p}_{k}$ has to be introduced. The closed formulation of the optimal solution is no longer applicable but a simple iterative algorithm can still find the best solution. The proof applies appropriately.

\section{Algorithm 1:}

1. Determine the required energy $E_{l}-E_{0}$

2. Sort the costs $c_{k}$ 
3. Iterate starting from the lowest cost: add up the energy per time step $c_{k} \bar{p}_{k} T$ until the required energy is exceeded.

4. Reduce the power $p^{\prime}$ for the last time step as required.

\section{Self-Discharge and Losses}

It is reasonable to assume that the charging efficiency is less than $100 \%$ and that the battery loses a certain part of its charge every time step. In general this leads to a new battery model:

$$
E_{k+1}=\alpha E_{k}-\beta+\gamma T p_{k}
$$

where $1-\alpha$ is the relative discharge coefficient for each time step, $\beta$ is the absolute discharge energy per time step and $\gamma$ is the charging efficiency. This changes the battery state of charge

$$
\text { calculation }
$$

to:

$$
E_{l}=\alpha^{l} E_{0}-\beta \sum_{k=0 \ldots l-1} \alpha^{k}+\gamma \sum_{k=0 \ldots l-1} \alpha^{l-k-1} T p_{k}
$$

A simple input transformation with $p_{k}^{\prime}=\frac{p_{k}}{\alpha^{l-k-1}}$ and

$$
E_{0}^{\prime}=\alpha^{l} E_{0}-\beta \sum_{k=0 \ldots l-1} \alpha^{k}
$$

can turn this new problem back into the problem discussed above. The transformation also applies to the charge power limit, which will therefore be time varying. The inverse transformation can be used to find the solution.

\section{Penalty on Final State of Charge}

Instead of making the final state of charge a fixed boundary condition, it is also possible to leave it variable but apply a cost penalty to any difference from the goal state. This would leak to an additional cost term

$$
J_{\text {penalty }}=\lambda\left(E_{l}-E_{\text {Goal }}\right)
$$

Again this new problem can easily be converted into the problem formulation above by adding an additional time step at the end where further charging at a cost equivalent to $\lambda$ is available to satisfy the original boundary condition.

\section{Resistive Losses}

Resistive losses within the battery (and the electricity supply) can be the dominating factor for charging losses. These resistive losses are proportional to the square of the current, which is approximately proportional to the square of the charging power:

$$
p_{\text {in }}=p_{\text {out }}+R p_{\text {out }}^{2}
$$

where $R$ is the resistance normalized for the charging power. In terms of the optimization problem, the losses can be Page 5 of 13 included either in the power going into the battery or in the cost of the electricity, depending on whether $p_{\text {in }}$ or $p_{\text {out }}$ is the wanted variable. The latter produces an easier problem definition:

$$
C_{k+1}=C_{k}+T c_{k}\left(p_{k}+R p_{k}^{2}\right)
$$

The solution to this extended problem lies in finding the admissible charge power inputs with the same differential cost $\frac{\partial J}{\partial p_{k}}=c_{k}\left(1+2 R p_{k}\right)$. This cost should be equal to the threshold cost $c^{\prime}$. The equation can be solved for the charging power $p_{k}=\frac{c^{\prime}-c_{k}}{2 R c_{k}}$, leading to the new control law:

$$
p_{k}^{*}=\left\{\begin{array}{c}
0, c_{k}>c^{\prime} \\
\frac{c^{\prime}-c_{k}}{2 R c_{k}}, c_{k}<c^{\prime} \text { and } \frac{c^{\prime}-c_{k}}{2 R c_{k}}<\bar{p} \\
\bar{p}, \text { otherwise }
\end{array}\right.
$$

Finding $c^{\prime}$ is more difficult now, because it is no longer equal to one of the $c_{k}$. Since the boundary condition $E_{l}$ is a monotonous function of $c^{\prime}$, it can be found using an iterative algorithm performing interval bisection.

\section{Allowing Discharge}

From the global perspective of grid stability, it would be desirable that a charging car feeds back electricity into the grid at times of high demand. Given the losses and regulatory difficulties involved this may not be an easy or profitable application to set up. Still, in exceptional circumstances (such as a grid in danger of collapse due to an unexpected electricity shortage), it would be advisable to do so.

In a way, formulating this new problem is as easy as extending the admissible values for charging power to negative values: $p_{k} \in[p, \bar{p}]$. Given some boundary conditions, this formulation is sufficient to find an admissible solution.

However, this problem formulation is missing an essential constraint: the lower limit of the battery state of charge. It was not previously relevant, but it could be violated by discharging an already empty battery. Another complicating factor is that the reward for feeding electricity back into the grid is different from the cost of taking electricity out of it - this is due to distribution charges and efficiencies.

For these reasons, the introduction of battery discharge breaks the temporal symmetry of the problem, and this leads directly to the more complex formulation detailed below.

\section{THE DYNAMIC CHARGING}

\section{PROBLEM}

Once process dynamics and the order of events have been taken into account, the charging problem becomes a lot more 
complex. The simple solution above was only possible because the order of steps did not matter, and this temporal symmetry lead to a simple optimal control law.

Without the temporal symmetry, the problem is similar to a model predictive control (MPC) problem, and depending on the assumption it can be linear, stochastic, or even non-linear. While standard solutions are available for the linear case, these do not apply to the stochastic or the non-linear case. The differences will be explained using a few typical problem formulations.

\section{Allowing Discharge}

As discussed above, allowing discharge seems like a trivial extension, but it requires the introduction of a state limit on the battery state of charge:

$$
E_{k} \in\left[0, E_{\text {full }}\right]
$$

This leads to a standard MPC problem. Typical solutions involve a discrete optimization to identify the time steps where limits apply.

\section{Stochastic Prices}

Real time electricity prices can change unexpectedly, so the assumption that they are known in advance is not a very practical one. A more realistic model uses a stochastic process to describe future prices. A standard first order linear process has this form where $z_{k}$ is an uncorrelated random variable with normal distribution.

$$
\begin{gathered}
c_{k+1}=a_{1} c_{k}+b_{1} z_{k}+b_{0} \\
E\left\langle z_{k}\right\rangle=0 \\
E\left\langle z_{k}^{2}\right\rangle=1
\end{gathered}
$$

The optimal solution, defined as providing the lowest expected $\operatorname{cost} E\langle J\rangle$, is very difficult to find. The standard MPC approach does not apply because the applicability of limits is no longer a discrete decision, but a stochastic event. The limits then turn the Gaussian probability distributions into piecewise Gaussian distributions, which are difficult to handle numerically.

This problem is of a much more complicated nature, because it asks the basic question whether it is better to charge at current electricity prices, or to wait for them to fall. The central question "are prices going up or down?" lies at the heart of economic markets and market theory, and it cannot be answered with certainty.

Typical solutions will be based on the Hamilton-JacobiBellman equation, which traces the expected cost based on a stochastic pricing model. Approximating and solving this equation is numerically challenging and it may not be practically feasible with any degree of accuracy. Theoretical advances are being made in a number of fields. Model Predictive Control (MPC) with stochastic weight models can be used [18] (most stochastic MPC approaches will consider only stochastic limits, not weights). On the other hand dynamic programming (usually involving quantization) and mixed integer algorithms can help to find the expected cost benefit of charging at specific times [19]. Finally there are a number of industry specific approaches coming from operations research, that deal with the question of optimal load shifting and scheduling using a limited capacity [20][21]. However, the complexity is significant, and while such approaches may be worthwhile for the management of large storage elements such as pumped-storage hydroelectric machines, they are not suitable for domestic applications at this point.

There is another interesting avenue: suboptimal solutions. Given that the price model is at best a simplification of the real market behavior, the problem formulation is not expected to be exact and there is little point in finding the optimal solution. Instead, a suboptimal approach offers the potential for a solution that is much easier to find and delivers most of the benefits of the optimal solution. There is a trade-off between computational complexity and error, and further studies are required to analyze this trade-off.

\section{Charge Dependency}

The last important class of extensions deals with charge dependent aspects. For example, a battery may age faster when fully charged or discharged, and this could be formulated as an additional non-linear cost term. Also the selfdischarge is typically higher at very high state of charge. Apart from breaking the temporal symmetry because of the state dependency, these issues also make the problem non-linear.

Non-linear MPC offers a way of solving this problem by using piece-wise linear functions to approximate the non-linearities, standard methods are again applicable.

\section{APPLICATION EXAMPLE: SMART METERS IN ILLINOIS}

\section{Smart Meters}

Smart meters are being rolled out in several places in the world, for example in Holland, Germany, and in the State of Illinois. Instead of the fixed tariff used by a conventional meter, smart meters can deal with frequently changing tariffs. Typically the cost of electricity charged changes every hour or half an hour, and the electricity company communicates either the current tariff or the expected tariff development for the next day to the customer.

The reason for introducing smart meters is that they encourage load shifting. This means that customers can move electricity 
intensive activities into periods where electricity is cheap and plentiful. This reduces the strain on the electricity grid both in terms of distribution and in terms of generation.

Once a significant share of electricity is generated from (generally uncontrollable) renewable sources, load shifting will be an important measure needed to align generation and consumption. However, little is known about consumer behavior. It is unclear whether small incentives are sufficient to change behavior in any significant way and it is also unclear whether there is a business case for automatic solutions.

This study tries to answer the second question, by calculating the potential benefit of a smart charging strategy for an electric vehicle in combination with a smart meter in Illinois.

\section{Assumptions}

The case study assumes a typical electric vehicle that is being used for a regular commute to work during the week and for reduced driving during the weekend.

The car is driven to work at 7am and driven back home at $5 \mathrm{pm}$. Charging is possible at home between $7 \mathrm{pm}$ and $7 \mathrm{am}$ using a smart meter. The electricity is provided by Ameren, and two tariffs are considered: the day-ahead tariff, where prices are set at $5 \mathrm{pm}$ for the following day and real time pricing. The prices for Ameren Illinois Zone have been taken from the Ameren web site [22] for the period from September $1^{\text {st }} 2011$ to September $1^{\text {st }} 2012$. These prices exclude the distribution cost, which is constant and therefore not relevant for comparison purposes.

Looking at the pricing information from a statistical point of view reveals a few surprises as shown in Table 2. First of all both the day-ahead price and even more so, the real time price become negative at times. The standard deviation of the realtime price is much higher than the day-ahead price. The difference between the day-ahead price and the real-time price can be seen as prediction error and interestingly its standard deviation is only slightly lower than standard deviation of the real-time price. The correlation coefficient between day-ahead prices and real time prices is 0.43 , which indicates that dayahead prices have only moderate value as a prediction of the real-time prices. Certainly the latter are much more volatile and therefore more interesting for load shifting.

Table 2: Statistic Properties of the Prices in cent

\begin{tabular}{|l|l|l|l|l|}
\hline & Mean & SD & Min & Max \\
\hline Day-Ahead & 2.73 & 1.04 & -0.19 & 19.81 \\
\hline Real Time & 2.63 & 2.20 & -8.85 & 107.58 \\
\hline Difference & -0.10 & 1.99 & -13.19 & 99.19 \\
\hline
\end{tabular}

Page 7 of 13
In addition, the following assumptions (Table 3) are used for the simulation. No specific vehicle is used as a reference, since electric vehicles are still at a very early stage. The GM Volt and the Nissan Leaf for example both have a smaller battery than assumed here (The Nissan Leaf has a battery with nominal $24 \mathrm{kWh}$ capacity but not all of that is actually usable.) Instead these figures are based on a slightly longer than average commute of about 35 miles one way, where the savings of an electric vehicle should be more pronounced than on a shorter commute.

Table 3: Assumed Constants

\begin{tabular}{|l|c|l|l|}
\hline Constant & Symbol & Value & Unit \\
\hline Usable Battery Capacity & $E_{\text {Total }}$ & 24 & $\mathrm{kWh}$ \\
\hline Weekday Consumption & $E_{W D}$ & 16 & $\mathrm{kWh}$ \\
\hline Weekend Consumption & $E_{W E}$ & 8 & $\mathrm{kWh}$ \\
\hline Charging Power & $P_{\text {Slow }}$ & 2 & $\mathrm{~kW}$ \\
\hline Charging Efficiency & $\eta$ & 90 & $\%$ \\
\hline Charging Period & $T_{\text {Charge }}$ & 12 & $\mathrm{~h}$ \\
\hline
\end{tabular}

\section{Charging Strategy}

The charging strategy is based on the basic optimal charging problem. The day-ahead prices are used as an indication for the electricity prices during the charging period, and the cheapest prices are used to charge the battery. The effect of this can be seen in Figure 1 - charging takes place during the hours of the night when the electricity has its lowest price. The detailed Simulink models used to create this simulation can be seen in Figure 5 and Figure 6.

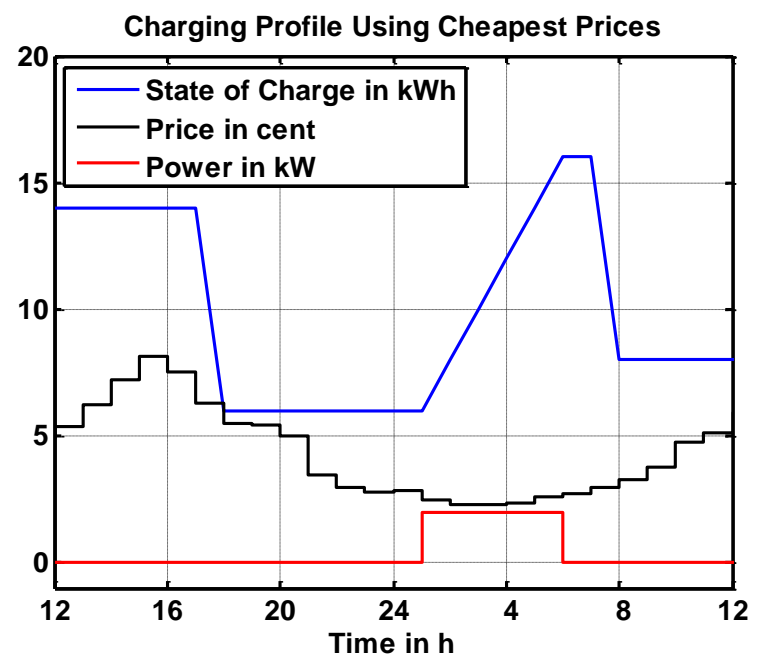

Figure 1: Charging Profile Overnight 
A number of different strategies and assumptions are simulated to test the effect on the overall electricity cost.

\section{RESULTS}

The car uses a total of $4928 \mathrm{~kW}$ during the simulated year and with the assumed $90 \%$ efficiency this means $5476 \mathrm{~kW}$ of electricity is used from the grid. With a traditional tariff at an average electricity price of 2.73 cent per $\mathrm{kWh}$, this would cost 149.4 USD.

\section{Charging Strategies}

The first graph compares the impact of the charging strategy on the electricity cost. For comparison purposes, two dumb strategies are considered first: charging as soon as the car is plugged in ("fixed early"), and charging as late as possible while still filling the battery before setting off ("fixed late").

The optimal strategy implements the selection of the cheapest tariffs while still filling the battery before setting off. For this purpose, a price prediction horizon of $24 \mathrm{~h}$ is used, of which only the $12 \mathrm{~h}$ covering the charging period are relevant.

As a variation, a second strategy looks ahead to the next night and decides whether it is cheaper to charge the battery fully or to fill the battery only as much as required for the daily commute, followed by an expected complete charge during the following night. Ideally this requires a prediction horizon covering two nights (48h), but this is not actually feasible because the prediction only extends to the end of the next day (indicated by a star in the graph). Therefore a realistic horizon of $31 \mathrm{~h}$ is also added to the comparison.

As can be seen in Figure 2, the annual electricity cost of charging an electric vehicle is highest when it is charged as soon as the owner gets home. Charging late in the morning (just in time) is more cost effective. Using an optimal charging strategy further reduces the cost and the benefit is increased very slightly by extending the prediction horizon.

\section{Real Time Pricing}

One issue with using day-ahead prices is that while the optimization algorithm is very simple, the price prediction is not very accurate. There are typically very significant differences between the prediction and the real time price of electricity, which means that using day-ahead prices is not providing the full benefit of load shifting to the individual and to the electricity grid.

As detailed above, the optimal charging problem for stochastic prices is both complicated and computational intensive, and no attempt is made here to provide an optimal solution. Instead, a number of approximations are pursued.
The first approximation is using the day-ahead price information to schedule the charging of the electric vehicle but in fact real time prices are used to calculate the cost ("RT Rate"). This can be achieved with minimal effort by changing electricity tariffs. In this case, the day-ahead price becomes effectively a disturbance model for the real time price development.

The second approximation uses the threshold cost $c^{\prime}$ as calculated using the day-ahead price information, but it compares it to the real time price of electricity to decide whether charging takes place or not ("RT Trigger"). Again this is simple to implement, although special care needs to be taken to ensure that the car always has sufficient charge at the beginning of the commute. The simulation does this by starting to charge irrespective of price if this is necessary to reach sufficient charge.

The final approximation assumes complete knowledge of real time prices ahead of time - otherwise it is identical to the dayahead optimization ("RT Optimal"). Obviously this is only possible to simulate in retrospect and it is not implementable because it uses knowledge of future events. But the simulation provides an upper limit for the potential savings possible using a price prediction model. It is worth noting that even using a optimal model, the savings may be significantly less than this upper limit.

It can be seen in Figure 2 that these algorithms provide significant reductions in cost. The more sophisticated the algorithm is, the bigger the savings. The effect of real time prices is distinctly more pronounced than the effect of different prediction horizons discussed before.

\section{Fast Charging}

All previous simulations are performed with a moderate charging power of $2 \mathrm{~kW}$, which is approximately the amount of power that can be provided by a standard electricity outlet. If a smart charger is used, it is reasonable to assume that it will be a dedicated fast charging unit, which can provide higher power levels. A higher charging power means a shorter charging duration and therefore load shifting is expected to become more effective.

To study this effect, charging powers of $2 \mathrm{~kW}, 4 \mathrm{~kW}$ and $8 \mathrm{~kW}$ are simulated using the charging strategies and pricing schemes introduced above. The same charging efficiency is assumed for all charging powers, which may not be quite realistic depending on battery technology. Although, when the infrastructure is advanced enough to support fast charging, the realistic powers could be higher than $10 \mathrm{~kW}$; it is important to remember that higher powers means a tradeoff between charging efficiency and price optimization.

The result shown in Figure 3 paints an interesting picture. First of all it is worth noting that fast charging is more expensive, if 
a bad fixed time charging strategy is being used. This is because the cheapest prices are typically found during the middle of the night and neither the early nor the late charging times make use of them. Especially charging once the vehicle is home can get quite expensive. Setting an early morning time for the start of charge, for example $3 \mathrm{am}$, provides much better results, leading to a cost of approximately 100 USD.

The next interesting conclusion is that fast charging does indeed provide better load shifting and a further reduction in electricity costs. The benefit depends on the charging strategy but it is in the order of 10 USD or more and it certainly is higher than the potential loss of efficiency due to the faster charging. Whether it is also high enough to compensate for the increased wear of the battery and the investment cost of the fast charger remains very much doubtful.

Finally the difference between the best feasible charging strategy ("RT Trigger") and the retrospect theoretical optimum ("RT Optimal") increases significantly with higher charging powers. It is only about $2.5 \mathrm{USD}$ at $2 \mathrm{~kW}$ but it increases to over $15 \mathrm{USD}$ at $8 \mathrm{~kW}$. This means that fast chargers create significant demand for better real time price prediction strategies.

Of course fast chargers may also provide an opportunity to perform load shifting on the faster time scales mentioned above. For example, they can help to absorb harmonics and noise to improve local power quality, or they could apply droop control to improve frequency stability of the electricity grid. But so far there is no business case for these measures and in fact it would cost the consumer both in terms of investment and in terms of loss of cheap electricity.

\section{SUMMARY/CONCLUSIONS}

The paper presented an optimization problem for the cost effective charging of electric vehicle. Assuming a day-ahead tariff, electricity prices are known in advance and a simple solution to this problem can be found and implemented. According to numerical simulations using real price information from Illinois, this leads to very moderate savings in the order of about 30 USD a year compared to immediate charging, and 10 USD compared to fixed time charging.

Larger savings can only be achieved by combining two measures: using a fast charger and changing to a real-time price tariff, where electricity prices are not known in advance. The problem of identifying the cheapest charging times becomes much more complicated in this case, because it depends on the prediction of future prices, which is not reliable. Using the same simple approach informed by dayahead price information, a reasonable solution can be found that saves another 20 USD. The potential for further savings in the order of 15 USD exists but it would rely on an operation model for predicting future electricity prices. It is questionable whether the effort for this would be worthwhile for a domestic application, however it might be beneficial if it is planned on a larger scale.

\section{REFERENCES}

[1] 2008, "World Energy Outlook 2008," IEA (International Energy Agency), Paris.

[2] Birol F., 2010, "World energy outlook 2010," IEA (International Energy Agency), Paris.

[3] Commission E., 2009, “Technology map: A European Strategic Energy Technology Plan (SET-Plan)," Joint Research Centre-European Commission, ....

COMMUNITIES C. O. T. E., 2008, "COMMUNICATION FROM THE COMMISSION TO THE COUNCIL, THE EUROPEAN PARLIAMENT, THE EUROPEAN ECONOMIC AND SOCIAL COMMITTEE, 2020 by 2020 Europe's climate change opportunity," European Union, pp. 1-12.

[5] Zubaryeva A., Thiel C., Barbone E., and Mercier A., 2012, "Assessing factors for the identification of potential lead markets for electrified vehicles in Europe: expert opinion elicitation," Technological Forecasting and Social Change, pp. 1-16.

2009, "World energy outlook 2009," IEA (International Energy Agency), Paris.

Kang J. E., and Recker W. W., 2009, “An activitybased assessment of the potential impacts of plug-in hybrid electric vehicles on energy and emissions using 1-day travel data," Transportation Research Part D: Transport and Environment, 14(8), pp. 541-556.

[8] 2009, "Nearly $20 \%$ of U.S. cars will be hybrids by 2020, forecast says," Automotive News.

[9] Allcott H., 2011, "Rethinking real-time electricity pricing," Resource and Energy Economics, 33(4), pp. 820-842.

[10] Pricing R. R., 2009, "Bringing Residential Real-Time Pricing to Scale in Illinois: Policy Recommendations," pp. 2-5.

[11] Heydt G., 1983, "The impact of electric vehicle deployment on load management strategies," IEEE Trans. Power Appar. Syst.;(United States), (5). 
[12] Webster R., 1999, "Can the electricity distribution network cope with an influx of electric vehicles?," Journal of Power Sources, 80(1-2), pp. 217-225.

[13] Camus C., and Farias T., 2011, "Impacts of electric vehicles' charging strategies in the electricity prices," 2011 8th International Conference on the European Energy Market (EEM), (May), pp. 833-838.

[14] Mahalik M., Poch L., Botterud A., and Vyas A., 2010, "Impacts of plug-in hybrid electric vehicles on the electric power system in Illinois," 2010 IEEE Conference on Innovative Technologies for an Efficient and Reliable Electricity Supply, pp. 341348.

[15] Acha S., Green T., and Shah N., 2011, "Optimal charging strategies of electric vehicles in the UK power market," Innovative Smart Grid ..., pp. 1-8.

[16] Kristoffersen T. K., Capion K., and Meibom P., 2011, "Optimal charging of electric drive vehicles in a market environment," Applied Energy, 88(5), pp. 1940-1948.

[17] Rotering N., Member S., and Ilic M., "Optimal Charge Control of Plug-In Hybrid Electric Vehicles In Deregulated Electricity Markets," pp. 1-9.

[18] Bemporad A., Puglia L., and Gabbrielline T., 2011, "A stochastic model predictive control approach to dynamic option hedging with transaction costs," American Control Conference, San Francisco, CA, USA, pp. 3862-3867.

[19] Thompson A. M., and Cluett W. R., 2005, "Stochastic iterative dynamic programming: a Monte Carlo approach to dual control," Automatica, 41(5), pp. $767-778$

[20] Kuznia L., Zeng B., Centeno G., and Miao Z., 2011, "Stochastic optimization for power system configuration with renewable energy in remote areas," Annals of Operations Research, (March), pp. 1-23.

[21] Schroeder A., Siegmeier J., and Creusen M., 2011, Demand management and storage sizing in electricity distribution grids.

[22] Corporation A., "Real Time Prices" [Online]. Available:

https://www2.ameren.com/RetailEnergy/realtimeprice s.aspx. [Accessed: 14-Sep-2012].

\section{CONTACT INFORMATION}

Please see http://smartinv.notlong.com for an overview of the research project and the people involved. The work has been conducted in the Department of Aeronautical and Automotive Engineering at Loughborough University.

http://www.lboro.ac.uk/departments/aae/

\section{ACKNOWLEDGMENTS}

Part of this work is supported by the UK government via EPSRC, the Engineering and Physical Sciences Research Council, under grant EP/E055877/1.

\section{DEFINITIONS/ABBREVIATIONS}

$\begin{array}{ll}\text { BEV } & \text { battery electric vehicle } \\ \text { DA } & \text { day-ahead pricing } \\ \text { HEV } & \text { hybrid electric vehicle } \\ \text { ICE } & \text { internal combustion engine } \\ \text { MPC } & \text { model (based) predictive control } \\ \text { RT/RTP } & \text { real time pricing } \\ \text { PHEV } & \text { plugin-in hybrid electric vehicle } \\ \text { SOC } & \text { (battery) state of charge } \\ \text { SD } & \text { standard deviation } \\ \text { V2G } & \text { vehicle to grid }\end{array}$




\section{APPENDIX}

\section{Cost Results}

\section{Day Ahead Pricing}

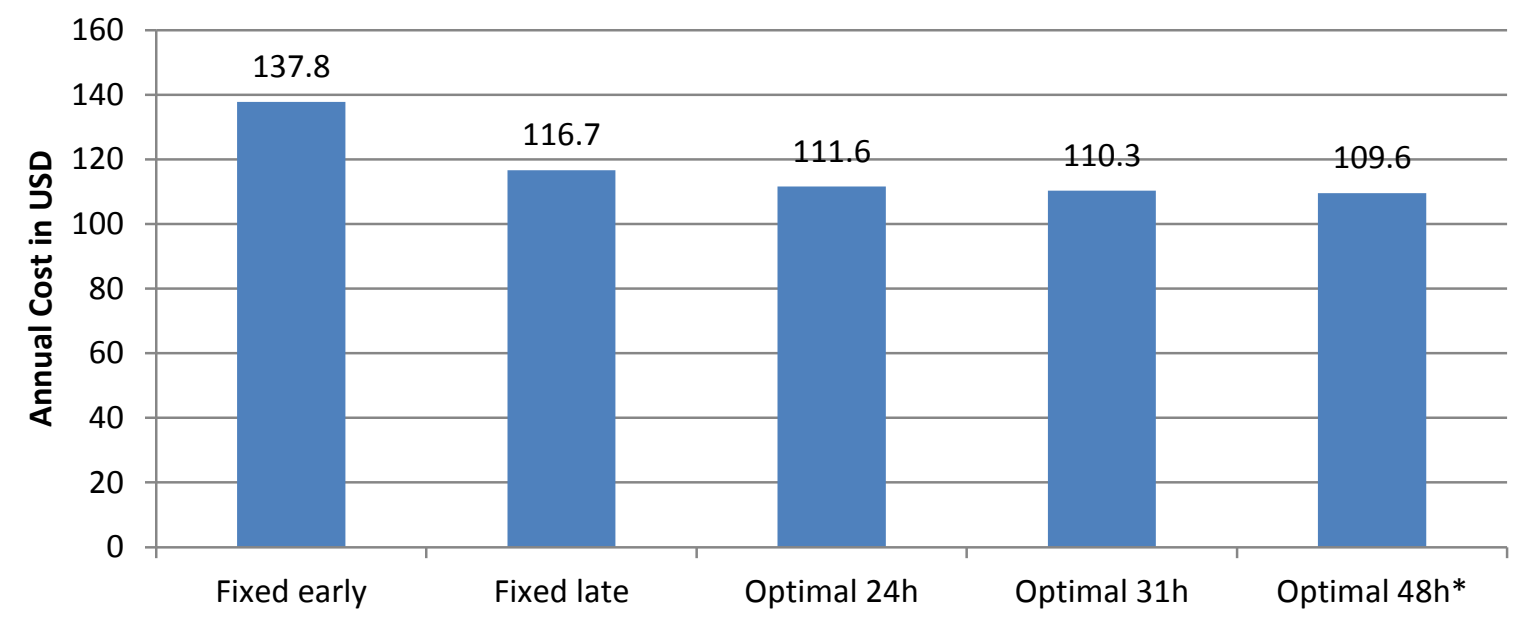

Day Ahead

Figure 2: Comparison of Charging Strategies Using Day-Ahead Pricing

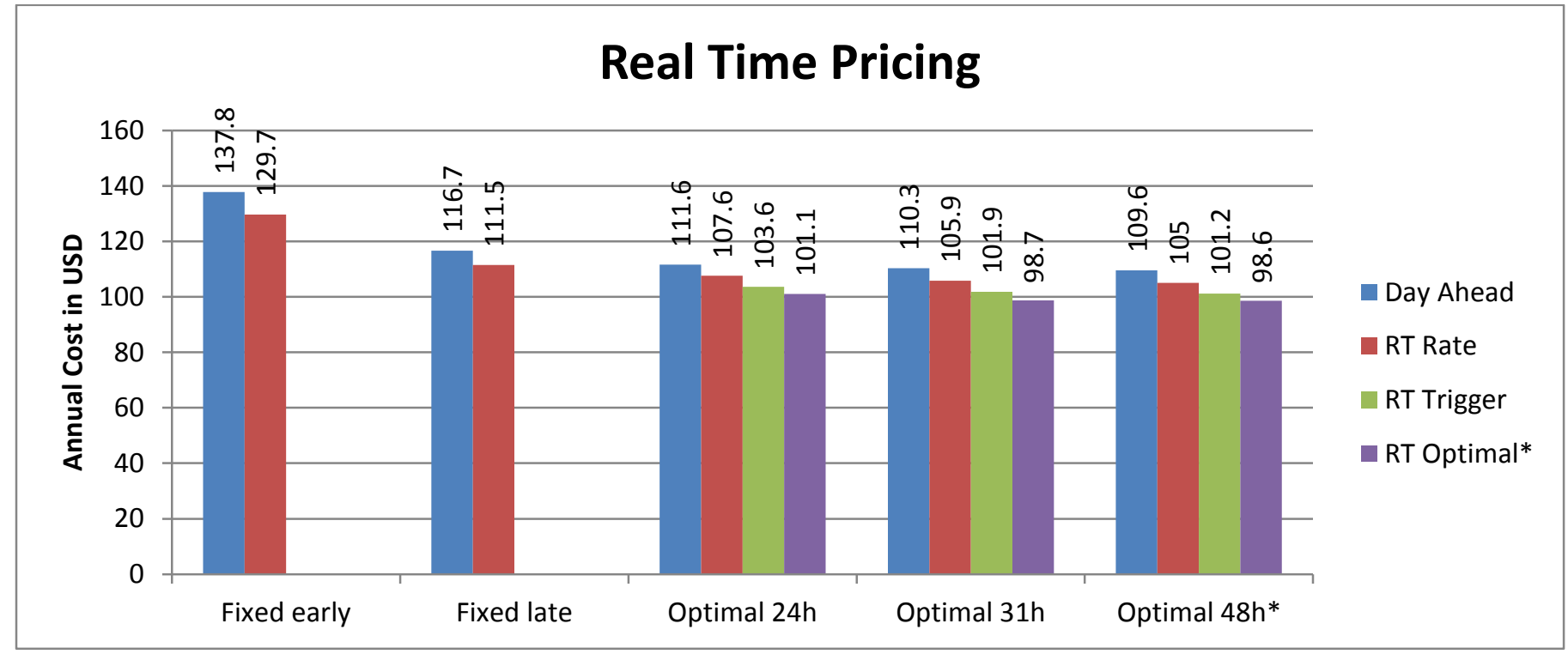

Figure 3: Comparison of Charging Strategies Using Real Time Pricing 


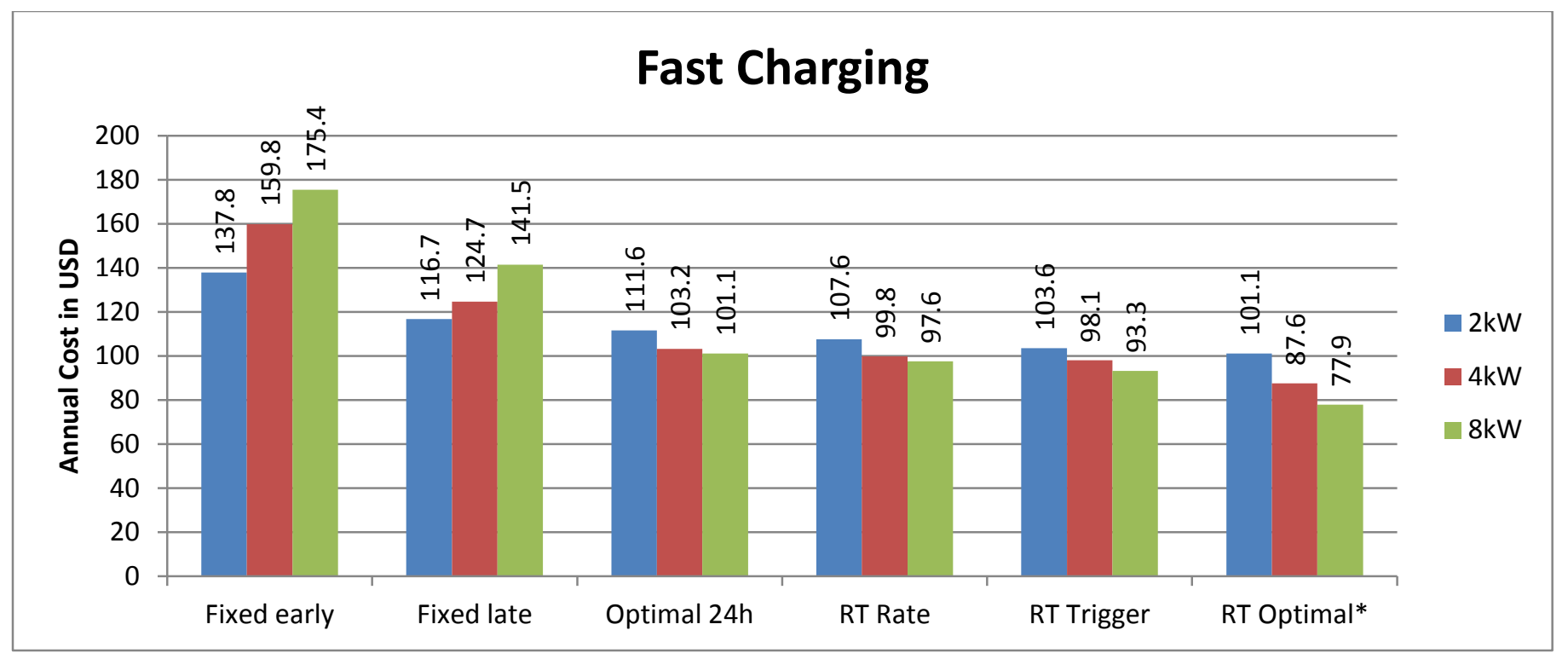

Figure 4: Comparison of Impact of Fast Charging for Different Strategies

\section{Simulation Model}

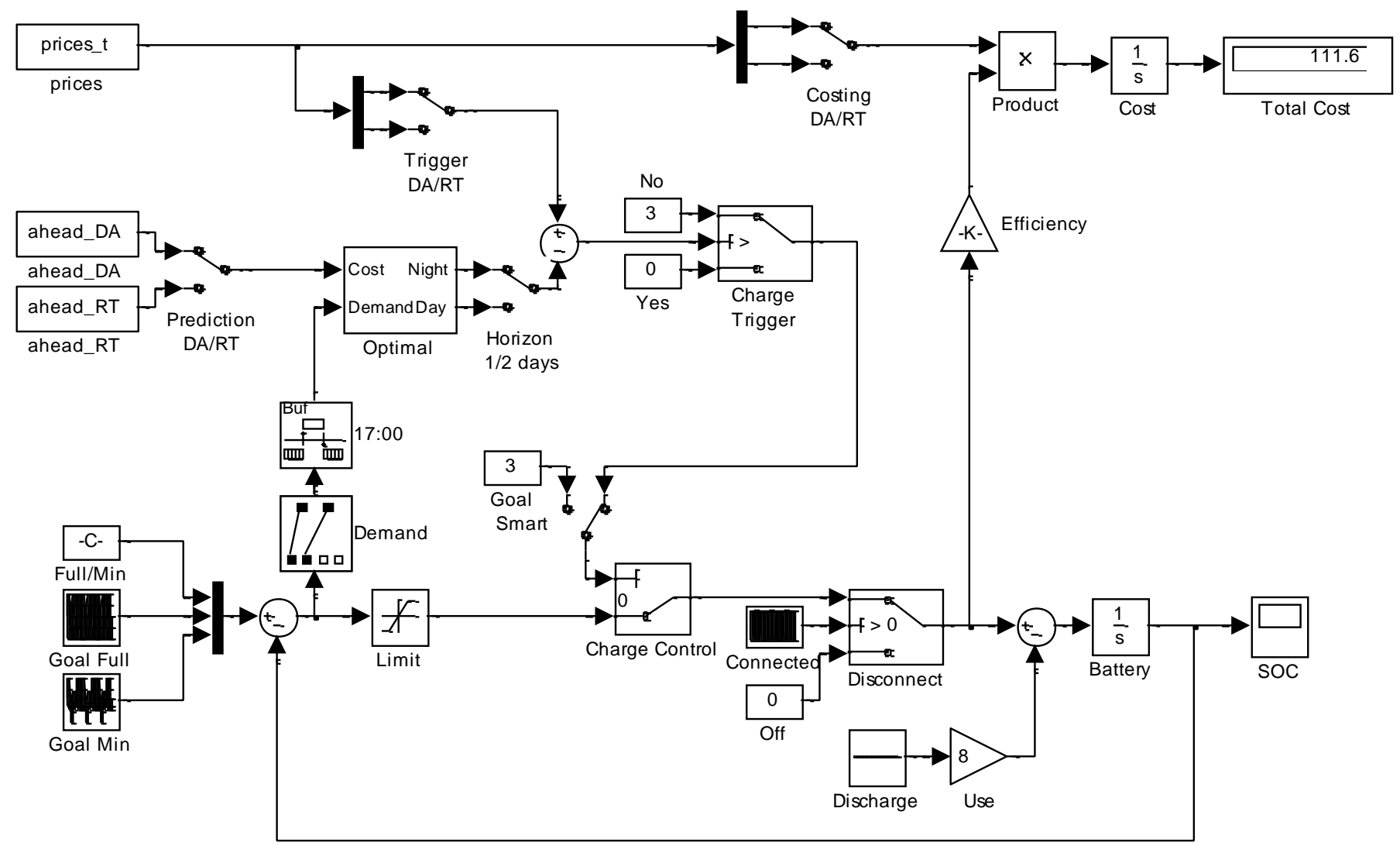

Figure 5: The Main Simulink Model 


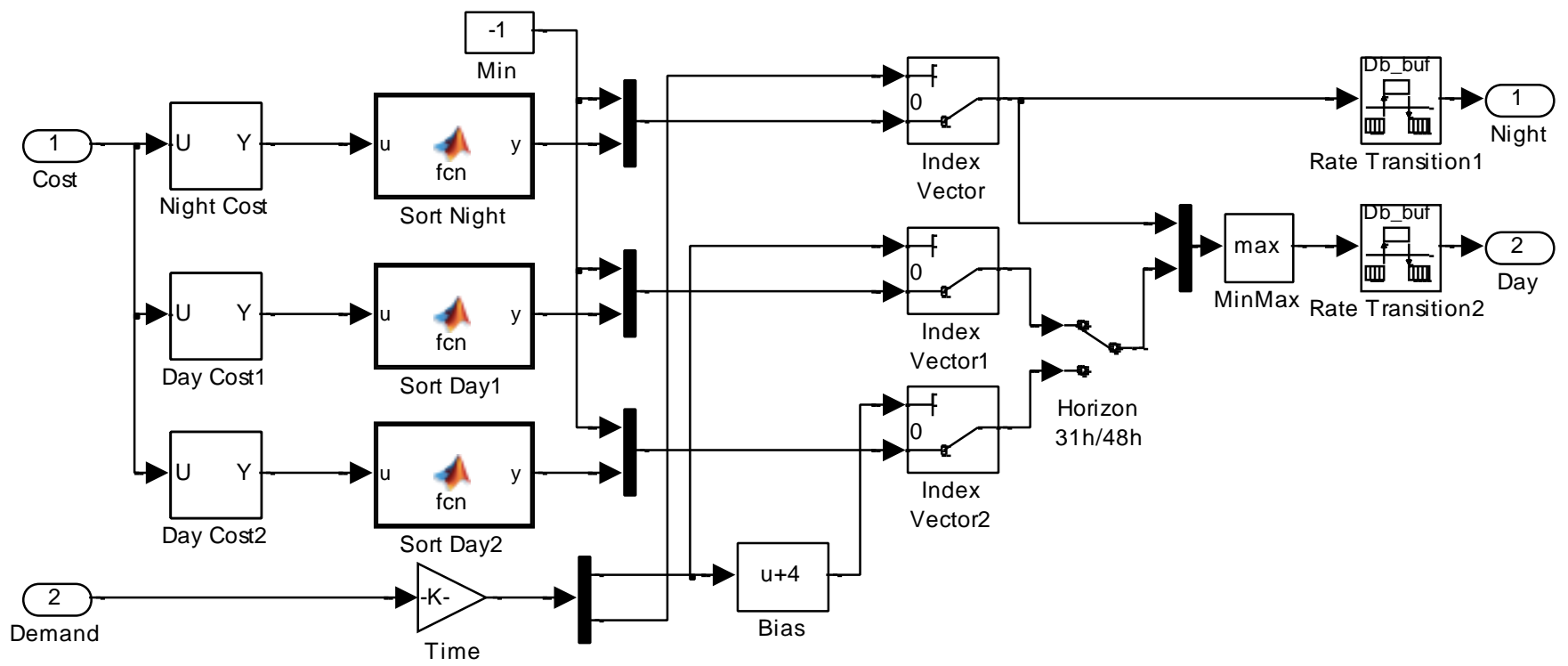

Figure 6: The Sub-Model with the Optimization Algorithm 